\title{
AN ATCA SURVEY OF DEBRIS DISKS AT 7 MILLIMETERS
}

\author{
L. Ricci ${ }^{1}$, S. T. Maddison ${ }^{2}$, D. Wilner ${ }^{1}$, M. A. MacGregor ${ }^{1}$, C. UbaCh $^{3}$, J. M. Carpenter $^{4}$, And L. Testi ${ }^{5}$ \\ ${ }^{1}$ Harvard-Smithsonian Center for Astrophysics, 60 Garden Street, Cambridge, MA 02138, USA; luca.ricci@cfa.harvard.edu \\ ${ }^{2}$ Centre for Astrophysics \& Supercomputing, Swinburne University, P.O. Box 218, Hawthorn, VIC 3122, Australia \\ ${ }^{3}$ National Radio Astronomy Observatory, North American ALMA Science Center, 520 Edgemond Road, Charlottesville, VA 22903, USA \\ ${ }^{4}$ Department of Astronomy, California Institute of Technology, MC 249-17, Pasadena, CA 91125, USA \\ ${ }^{5}$ European Southern Observatory (ESO) Headquarters, Karl-Schwarzschild-Str. 2, D-85748 Garching bei Muenchen, Germany \\ Received 2015 September 4; accepted 2015 October 12; published 2015 November 9
}

\begin{abstract}
We present Australia Telescope Compact Array continuum observations at a wavelength of $6.8 \mathrm{~mm}$ of five debris disks: $\beta$ Pictoris, $\mathrm{q}^{1}$ Eridani, HD 107146, HD 181327, and HD 95086. These observations provide the detection at the longest wavelengths obtained to date for all these debris disks. By combining our $6.8 \mathrm{~mm}$ data with previous detections at shorter sub-millimeter/millimeter wavelengths we measure the long wavelength spectral index of these sources. We then use previous estimates for the temperature of the emitting dust to derive the spectral index of the dust emissivity. Under the assumption that all the detected flux comes from dust only, we constrain the slope of the solid size distribution, assumed to be a power-law. The values that we infer for the slope of the size distribution range between about 3.36 and 3.50. We compare our findings with the case of the Fomalhaut debris disk and use these results to test the predictions of collisional cascades of planetesimal belts.
\end{abstract}

Key words: circumstellar matter - planets and satellites: formation - stars: individual (HD107146) submillimeter: stars

\section{INTRODUCTION}

Observations of debris disks made of cold dust around nearby stars provide crucial information to the process of planet formation (e.g., Zuckerman 2001; Matthews et al. 2014). The dust grains observed in these systems are thought to be produced by collisions between $\mathrm{km}$-sized planetesimals (comets and asteroids), leftovers of the planetary formation process (see Wyatt 2008). The highly destructive mutual encounters between planetesimals which generate the observed dust are probably triggered by the dynamical interaction with one or more planets in the system (Mustill \& Wyatt 2009).

Because of their extremely faint emission, $\mathrm{km}$-sized planetesimals cannot be directly observed outside the solar system. However, information on their physical and dynamical properties can be extracted from the size distribution of the emitting smaller dust grains. Observations in the sub-millimeter/ millimeter can quantify the departure of the spectrum from the Rayleigh-Jeans regime $\left(\propto \nu^{2}\right)$ which is due to a combination of temperature and emissivity effects. As the temperature of dust grains can be inferred from observations in the infrared, the frequency-dependence of the dust emissivity at sub-mm/ $\mathrm{mm}$ can be estimated, and this is related to the size distribution of $\sim 1-10 \mathrm{~mm}$-sized dust grains present in a debris disk (Draine 2006; Gaspar et al. 2012; Ricci et al. 2012). Observations at long wavelengths have the advantage of minimizing the departures from the Rayleigh-Jeans approximation as well as the effect of optical depth because of the lower dust emissivities (e.g., Testi et al. 2014).

This method can be used to test collisional models of debris disks which provide different predictions for the solid size distribution depending on the dynamical state (e.g., velocity dispersion, Pan \& Schlichting 2012) or physical conditions (e.g., tensile strength, Durda \& Dermott 1997; Gaspar et al. 2012) of the large colliding bodies. The reference model is the collisional cascade of large bodies at the steady state, which predicts a power-law solid size distribution $\left(n(a) \propto a^{-q}\right)$ with exponent $q=3.51$, as a result of the collective dynamical interaction of particles caused by inelastic collisions and fragmentation (Dohnanyi 1969). This standard model assumes a single constant tensile strength ${ }^{6}$ and velocity dispersion for all bodies regardless of size. More recent numerical simulations and analytical calculations have relaxed these assumptions and derived steeper grain size distributions $(q \gtrsim 3.5-3.6)$ in the case in which either the tensile strength or the velocity dispersion of the large bodies are steep functions of their size (Gaspar et al. 2012; Pan \& Schlichting 2012).

We present the results from observations with the Australia Telescope Compact Array ${ }^{7}$ (ATCA) of five debris disks at $6.8 \mathrm{~mm}: q^{1}$ Eri, HD 107146, HD 181327, HD 95086, $\beta$ Pic. In the case of $\beta$ Pic, the ATCA observations were already introduced in Wilson et al. (2011). We combine these data with previous observations at shorter wavelengths to estimate the $q$ values of the slope of the size distribution for each debris disk in our sample, and compare our results with the predictions of the collisional cascade models.

In Section 2 we present the main properties of the debris disks in our sample. Sections 3 and 4 describe the ATCA observations and their results, respectively. Results on the slope of the solid size distribution are presented and discussed in Sections 5 and 6. Section 7 summarizes the main findings of this study.

\section{THE SAMPLE}

In this section we present the main properties of the five debris disks in our sample. These sources were selected to be (1) at declinations lower than $20^{\circ}$ in order to be observable with the ATCA array, (2) relatively bright sources in the sub-mm,

\footnotetext{
6 The tensile strength of a body is defined as the minimum energy per unit mass required to disrupt it catastrophically.

7 The Australia Telescope Compact Array is part of the Australia Telescope National Facility which is funded by the Commonwealth of Australia for operation as a National Facility managed by CSIRO.
} 
i.e., with a flux density at $0.85 \mathrm{~mm}$ greater than $30 \mathrm{mJy}$, and (3) relatively compact, i.e., with angular size lower than $15^{\prime \prime}$ to maximize surface brightness sensitivity.

\section{1. $q^{1}$ Eri}

$\mathrm{q}^{1}$ Eridani (HD 10647) is an F9 main sequence star at a distance of $17.4 \mathrm{pc}$. Its estimated age is $\approx 1.7-3.2 \mathrm{Gyr}$ (Bonfanti et al. 2015; Moro-Martin et al. 2015). $\mathrm{q}^{1}$ Eri hosts $\mathrm{a} \sim 1 M_{\text {Jup }}$ planet which was discovered with the radial velocity technique at a distance of $\approx 2 \mathrm{AU}$ from the star (see Butler et al. 2006).

Evidence for a dusty belt has been obtained from observations in the infrared (Zuckerman \& Song 2004; Chen et al. 2006; Trilling et al. 2008) and sub-millimeter (Liseau et al. 2008). Recently, Moro-Martin et al. (2015) have fitted the disk Spectral Energy Distribution (SED) including far-IR fluxes with the Herschel Space Observatory. They inferred a dust temperature of $\approx 49 \mathrm{~K}$ at a stellocentric distance of about $40 \mathrm{AU}$.

\section{2. $H D 107146$}

HD 107146 is a solar-like G2 type star. At a distance of $27.5 \mathrm{pc}$ and with an age of $\sim 80-200 \mathrm{Myr}$, this is the brightest known debris disk at infrared wavelengths around a G-type star (Silverstone 2000; Moor et al. 2006). The disk was spatially resolved both in scattered light with the Hubble Space Telescope (Ardila et al. 2004; Ertel et al. 2011; Schneider et al. 2014) and in dust thermal emission with sub-millimeter single dish (Williams et al. 2004) and interferometric observations (Corder et al. 2009; Hughes et al. 2011; Ricci et al. 2015).

Spitzer spectroscopic and photometric data revealed the presence of warm dust $(\sim 120 \mathrm{~K})$ located $\sim 5-15 \mathrm{AU}$ from the star (Morales et al. 2011). A colder dust component extending between stellocentric radii of about 30 and $150 \mathrm{AU}$ dominates the sub-mm emission (Ricci et al. 2015). Recent observations with the Atacama Large Millimeter Array (ALMA) show a decrease in the surface brightness of the disk at $\approx 80 \mathrm{AU}$ from the star, which could be due to dynamical interaction with a low-mass terrestrial planet in the system (Kenyon \& Bromley 2015; Ricci et al. 2015).

\section{3. $H D 181327$}

HD181327 is an F6-type star at a distance of $\approx 51 \mathrm{pc}$ (Zuckerman \& Song 2004; Casagrande et al. 2011). It is located in the $\beta$ Pic moving group with an age of $23 \pm 3 \mathrm{Myr}$ (Mamajek \& Bell 2014).

In order to reproduce the emission in the infrared, Mittal et al. (2015) invoked a warm dust component at a stellocentric distance of $\approx 0.3-2 \mathrm{AU}$ (temperature of $\approx 340 \mathrm{~K}$ ), as well as a colder component with $\approx 82 \mathrm{~K}$ further from the star. Schneider et al. $(2006,2014)$ presented spatially resolved observations of the debris disk in scattered light at optical and near-infrared wavelengths with the Hubble Space Telescope. These observations show that a relatively narrow ring at a distance of $\approx 88 \mathrm{AU}$ from the star accounts for most of the total flux from the disk.

\section{4. $H D 95086$}

HD 95086 is an A8-type member of the Lower Centaurus Crux Association at a distance of $\approx 90 \mathrm{pc}$ (de Zeeuw et al. 1999; van Leeuwen 2007) and age of $17 \pm 6$ Myr. A planet with a mass of $5 \pm 2 M_{\text {Jup }}$ has been directly imaged at a projected distance of $56 \mathrm{AU}$ from the star (Rameau et al. 2013).

The observed disk SED of HD 95086 is best described with two dust temperatures: a warm component at $\approx 175 \mathrm{~K}$ and a colder component at $\approx 55 \mathrm{~K}$ with an outer radius of $\sim 200 \mathrm{AU}$ (Su et al. 2015). The analysis of spatially resolved images in the far-infrared indicates the presence of dust grains at further distances, possibly a diffuse halo of small particles thrown into highly eccentric or hyperbolic orbits by stellar radiation pressure.

\section{5. $\beta$ Pic}

$\beta$ Pictoris (HD 39060) is an A6 type star at a distance of $19.4 \mathrm{pc}$ and with an age of $23 \pm 3 \mathrm{Myr}$ (Gray et al. 2006; van Leeuwen 2007; Mamajek \& Bell 2014).

The star is surrounded by the first debris disk to be imaged in scattered light (Smith \& Terrile 1984). Subsequent observations have shown the existence of a massive planet at $\sim 10 \mathrm{AU}$ from the star (Lagrange et al. 2010), comets falling toward the star within a few AU (Vidal-Madjar et al. 1994), atomic gas extending out to $\sim 300 \mathrm{AU}$, sub-mm dust emission out to $\approx 100 \mathrm{AU}$ (Wilner et al. 2011), as well as a clear azimuthal asymmetry in the spatial distribution of molecular gas (Dent et al. 2014).

\section{ATCA OBSERVATIONS AND DATA REDUCTION}

ATCA observations of the $6.8 \mathrm{~mm}$ dust continuum toward the five disks in our sample were obtained using the Compact Array Broadband Backend (CABB) digital filter bank (Wilson et al. 2011).

Observations were carried out on 2009 September 14 for $\beta$ Pic (see Wilson et al. 2011 for a presentation of these observations), and between 2012 July 27 and August 5 for the other four disks. The ATCA array was in the H214 configuration for the observations of $\beta$ Pic (baselines between 82 and $247 \mathrm{~m}$ ), and in the $\mathrm{H} 75$ configuration for the other observations (baselines between 31 and $89 \mathrm{~m}$ ). The array comprises six $22 \mathrm{~m}$ diameter antennas, but data from the stationary sixth antenna, located about $6 \mathrm{~km}$ from the others, was discarded because of the higher phase noise on the much longer baselines. To obtain the best sensitivity in the continuum the correlator was set to cover the full $4 \mathrm{GHz}$ CABB effective bandwidth. The mean frequency of the observations is $44.0 \mathrm{GHz}$, corresponding to a wavelength of about $6.8 \mathrm{~mm}$.

Table 1 summarizes the coordinates of each target, the onsource times and gain calibrators. We used 0537-441, 1253055, and 1921-293 as bandpass calibrators, whereas Uranus, Neptune, and 1934-638 were used to calibrate the absolute flux scale of the data. We adopt the typical value of $10 \%$ for the uncertainty on the absolute flux scale for ATCA observations at this wavelength (e.g., Ubach et al. 2012). This uncertainty is in agreement with the variation of the flux for the gain calibrators as measured in different days during our observations.

The ATCA data were calibrated using the miriad software package. Imaging and deconvolution were performed with the standard miriad routines invert, clean, and restore. 
Table 1

ATCA Observations

\begin{tabular}{lcccc}
\hline \hline Source & R.A. & Decl. & $\begin{array}{c}\text { On-source } \\
\text { Time } \\
\text { (minutes) }\end{array}$ & $\begin{array}{c}\text { Gain Cali- } \\
\text { brator(s) }\end{array}$ \\
\hline $\mathrm{q}^{1}$ Eri & $01: 42: 29.32$ & $-53: 44: 27.0$ & 488 & $0208-512$ \\
HD 107146 & $12: 19: 06.50$ & $+16: 32: 53.9$ & 490 & $1236+077$ \\
HD 181327 & $19: 22: 58.94$ & -543217.0 & 721 & $1933-400$, \\
HD 95086 & $10: 57: 03.02$ & $-68: 40: 02.5$ & 712 & $1941-554$ \\
$\beta$ Pic & $05: 47: 17.09$ & $-51: 03: 59.4$ & 680 & J1147-6753 \\
\hline
\end{tabular}

\section{RESULTS OF THE ATCA OBSERVATIONS}

Continuum emission from all the five disks was detected with our ATCA observations at $6.8 \mathrm{~mm}$ (Table 2). The achieved signal-to-noise ratios range between $\approx 4$ (HD 95086) and $\approx 12$ (HD 181327).

As expected, the emission is spatially unresolved for the four disks observed in the $\mathrm{H} 75$ array configuration ( ${ }^{1}$ Eri, HD 107146, HD 181327, HD 95086), as the angular resolution for these observations is significantly larger than the angular extent of those sources. For these four spatially unresolved disks, the flux density was extracted using the uvfit routine that fits the calibrated visibilities with a point-source model.

In the case of $\beta \mathrm{Pic}$, the higher angular resolution achieved with the $\mathrm{H} 214$ array configuration allowed us to spatially resolve the disk (Figure 1). The disk is elongated along the northeast-southwest direction, as seen in all the previous maps for this source at shorter wavelengths. The map at $6.8 \mathrm{~mm}$ also shows higher surface brightness toward the southwest lobe of the disk, as previously seen at $0.87 \mathrm{~mm}$ (Dent et al. 2014) and $1.3 \mathrm{~mm}$ (Wilner et al. 2011) with better signal-to-noise. For this disk, the total flux density reported in Table 2 was obtained by integrating the surface brightness over the area showing emission at $\gtrsim 2 \sigma$ above the background level in the map.

\section{THE SLOPE OF THE SOLID SIZE DISTRIBUTION}

Models of collisional cascades for planetesimal belts predict size distributions which are well approximated by power-laws $n(a) \propto a^{q}$, typically with $3<q<4$, and between blow-out grain sizes (typically of the order of $\sim \mu \mathrm{m}$ ) and the sizes of the solids which initiate the cascade, i.e. $\sim 1-100 \mathrm{~km}$ (e.g., Dohnanyi 1969).

Under these conditions, Draine (2006) derived a relation between the spectral index $\beta$ of the dust emissivity $\left(\kappa_{\nu} \propto \nu^{\beta}\right)$, the spectral index $\beta_{\mathrm{s}}$ of small particles only (i.e., with sizes much smaller than the observing wavelengths), and the powerlaw index $q$ of the solid size distribution. This formula reads: $\beta=\beta_{\mathrm{s}}(q-3)$.

Since the dust thermal emission in debris disks is optically thin, the flux density $F_{\nu} \propto B_{\nu}\left(T_{\text {dust }}\right) \kappa_{\nu} \propto B_{\nu}\left(T_{\text {dust }}\right) \nu^{\beta}$, where $B_{\nu}\left(T_{\text {dust }}\right)$ is the Planck function evaluated at the temperature of the dust in the disk. For debris disks around stars with spectral types of $\mathrm{G}$ and earlier, such as in our sample, the dust is usually warm enough $\left(k_{B} T_{\text {dust }} \gg h \nu\right)$ for the Planck function to be well approximated by the Rayleigh-Jeans form $\left(B_{\nu}\left(T_{\text {dust }}\right) \propto \nu^{2}\right)$. A more accurate approximation is obtained considering the Planck function as a power-law, $B_{\nu}\left(T_{\text {dust }}\right) \propto \nu^{\alpha \text { Planck }}$ with $\alpha_{\text {Planck }}=\alpha_{\text {Planck }}\left(T_{\text {dust }}\right) \lesssim 2$, and equal to 2 in the Rayleigh-Jeans regime.
The flux density of debris disks at sub-mm and mm wavelengths is a power-law in frequency $\left(F_{\nu} \propto \nu_{\mathrm{mm}}^{\alpha}\right)$, and Draine's formula can be written in terms of $q$ as

$$
q \approx \frac{\alpha_{\mathrm{mm}}-\alpha_{\text {Planck }}}{\beta_{\mathrm{s}}}+3
$$

(see Ricci et al. 2012).

For $\beta_{\mathrm{s}}$ we consider a value of $1.8 \pm 0.2$, consistent with observations of small dust grains in several diffuse interstellar clouds, molecular clouds, as well as with predictions for small grains with chemical compositions expected in protoplanetary disks (e.g., Draine 2006; Testi et al. 2014, and references therein). Hence, by measuring the sub- $\mathrm{mm} / \mathrm{mm}$ spectral index $\alpha_{\mathrm{mm}}$ and by inferring $\alpha_{\text {Planck }}$ from the dust temperature estimated for each disk, we can use Equation (1) to infer the slope $q$ of the solid size distribution. ${ }^{8}$

Table 3 lists the flux density of each disk at a sub-mm/mm wavelength $\lambda_{1}$ reported in the literature which is combined with our ATCA flux density at $6.8 \mathrm{~mm}$ to measure $\alpha_{\mathrm{mm}}$ $\left(=\log \left(F_{\nu, \lambda_{1}} / F_{\nu, \lambda=6.8 \mathrm{~mm}}\right) / \log \left(\lambda_{1} / 6.8 \mathrm{~mm}\right)\right)$, the dust temperature $T_{\text {dust }}$, the spectral index of the Planck function $\alpha_{\text {Planck }}$ $\left(=\log \left(B_{\nu, \lambda_{1}} / B_{\nu, \lambda=6.8 \mathrm{~mm}}\right) / \log \left(\lambda_{1} / 6.8 \mathrm{~mm}\right)\right)$, and the slope of the solid size distribution $q$ as derived using Equation (1). For HD 107146 and $\beta$ Pic we chose the accurate flux measurements extracted from published ALMA observations. For the other three disks for which ALMA observations have not been obtained yet, we chose flux measurements with Herschel $\left(q^{1}\right.$ Eri, HD 95086) and APEX (HD 181327), see references in Table 3. In the cases in which Herschel and APEX observations are both present, we have verified that the $q$-values obtained using either one or the other flux density are consistent with each other at the $\lesssim 2 \sigma$ level.

For $\beta$ Pic, the sub-mm $/ \mathrm{mm}$ flux reported in the table was extracted from ALMA observations at $0.87 \mathrm{~mm}$ (Dent et al. 2014). Like our ATCA observations for the same source, also the ALMA observations were affected by spatial filtering of the emission on the largest scales. The shortest projected baselines of the ALMA have $u v$-distances of $17 \mathrm{k} \lambda$. In order to compare emission on the same spatial scales, we derived the emission including only visibilities associated to $u v$-distances of $17 \mathrm{k} \lambda$ and larger from our ATCA dataset. We derived a value of $185 \pm 33.6 \mu \mathrm{Jy}$ (rms noise on the map is $28.0 \mu \mathrm{Jy} /$ beam). We used this value to derive the spectral index $\alpha_{\mathrm{mm}}$, and associated uncertainty, reported in Table 3.

In all cases but HD 107146, the dust temperature $T_{\text {dust }}$ was inferred from fitting of the SED at infrared and sub-mm wavelengths. A reasonable uncertainty of $\pm 10 \mathrm{~K}$ was considered to derive an uncertainty on $\alpha_{\text {Planck }}$. In the case of HD 107146, we considered the interval of temperatures for dust with size of $\approx 1 \mathrm{~mm}$ at the stellocentric radii of $\approx 30-150 \mathrm{AU}$, as estimated by Ricci et al. (2015). Note that this interval includes the estimate of $\approx 51 \mathrm{~K}$ as derived through SED fitting by Williams et al. (2004). In this case, the range of dust temperatures considered here, i.e., $28 \mathrm{~K}$, is broader than the range considered for the other disks, i.e., $20 \mathrm{~K}$, because of the wider range of radial distances found for the unusually broad disk surrounding HD 107146 (Ricci et al. 2015). The dependence of $\alpha_{\text {Planck }}$ on the dust temperature is shown for each disk in Figure 2.

\footnotetext{
8 Here we are implicitly assuming that all the emission observed at sub-mm/ $\mathrm{mm}$ wavelengths comes from dust. The validity of this assumption will be discussed at the end of Section 6 .
} 
Table 2

Results of the ATCA Observations

\begin{tabular}{|c|c|c|c|c|c|}
\hline Source & Beam Size & $\begin{array}{l}\text { Beam P. A. } \\
\text { (degrees) }\end{array}$ & $\begin{array}{l}\text { Flux Density at } 6.8 \mathrm{~mm} \\
\qquad(\mu \mathrm{Jy})\end{array}$ & $\begin{array}{l}\text { rms Noise } \\
(\mu \mathrm{Jy} / \text { beam })\end{array}$ & $\begin{array}{c}\text { Total Uncertainty of Flux Density } \\
(\mu \mathrm{Jy})\end{array}$ \\
\hline $\mathrm{q}^{1}$ Eri & $14 " .81 \times 10$ ". 32 & 278.4 & 92.6 & 13.8 & 16.6 \\
\hline HD 107146 & $12 " .37 \times 10.77$ & 0.1 & 166.0 & 19.0 & 25.2 \\
\hline HD 181327 & $13 ! .24 \times 9 ! .82$ & 277.4 & 145.0 & 12.6 & 19.2 \\
\hline HD 95086 & $12 " .68 \times 11 " .04$ & 284.9 & 61.9 & 14.6 & 15.9 \\
\hline$\beta \mathrm{Pic}$ & $4 ! .18 \times 3 ! .56$ & 68.7 & $240.0^{\mathrm{c}}$ & 23.0 & 33.2 \\
\hline
\end{tabular}

Notes.

a The beam Position Angle is defined as the angle east of north to the disk major axis.

b The total uncertainty of flux density includes the rms noise and the uncertainty on the calibration of the absolute flux scale.

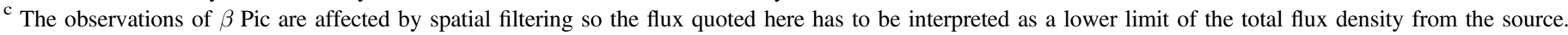

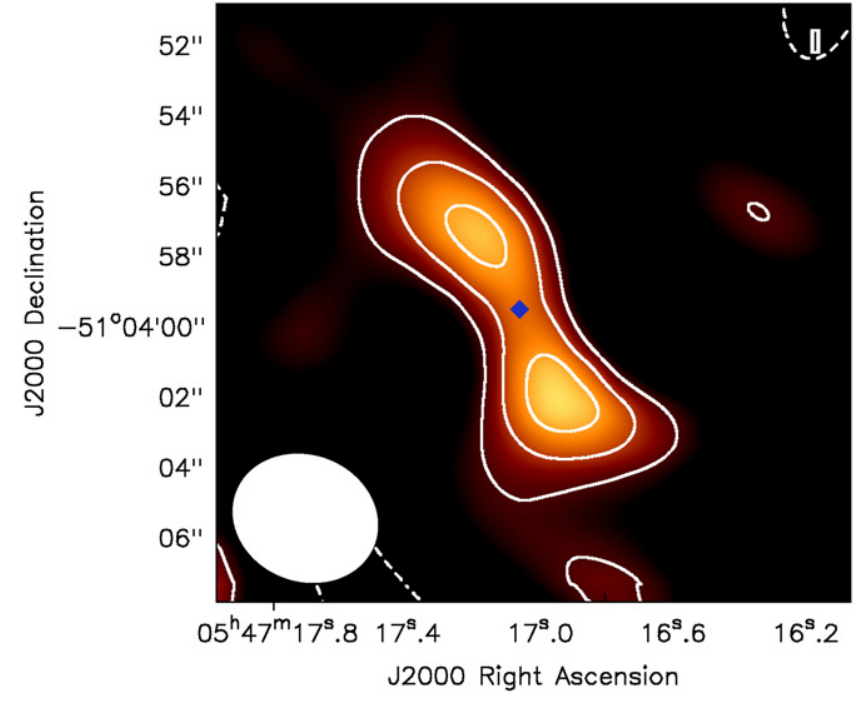

Figure 1. ATCA $6.8 \mathrm{~mm}$ continuum map of $\beta$ Pic. Contours are drawn at $-2 \sigma$ (dashed lines), $2 \sigma, 3 \sigma, 4 \sigma$ (solid), where $1 \sigma=23.0 \mu \mathrm{Jy} / \mathrm{beam}$ is the rms noise of the map. The blue symbol toward the center of the map shows the location of the star. The white ellipse in the lower left corner represents the synthesized beam with FWHM size of 4 !. $18 \times 3$ !. 56 and a position angle of $68^{\circ} .7$.

In Table 3 , the uncertainty on $q$ comes from the propagation of the uncertainties on $\alpha_{\mathrm{mm}}, \alpha_{\text {Planck }}$ and $\beta_{\mathrm{s}}$ using Equation (1).

\section{DISCUSSION}

In the previous section we have estimated the slope $q$ of the solid size distribution for our five debris disks. Values range between 3.36 (HD 107146) and 3.50 (HD 181327) with uncertainties of $0.06-0.08$. Using the same analysis described here, Ricci et al. (2012) obtained $q=3.48 \pm 0.11$ in the case of Fomalhaut. ${ }^{9}$ The $q$-values derived for the different disks are consistent within the uncertainties, with a weighted mean $\langle q\rangle=3.42 \pm 0.03$. Note however that debris disks may in principle have different values for $q$.

These values can be used to test predictions of models of collisional cascades. The typical benchmark model is the classical Dohnanyi (1969) prediction of $q=3.51$ for a collisional cascade at the steady-state as due to the collective dynamical interaction of particles caused by inelastic collisions and fragmentation. This model assumes that neither the

\footnotetext{
9 Note that the uncertainty on the value reported here is slightly different than the one reported in Ricci et al. (2012) because of a better treatment of the propagation of the uncertainties.
}

velocity dispersion of bodies nor their tensile strength is a function of the size of the bodies.

Pan \& Schlichting (2012) have extended the Dohnanyi (1969) treatment by assuming that the velocity dispersion and tensile strength vary with the size of the bodies as power-laws. They found self-consistent steady-state solutions when viscous stirring balances velocity damping. In these models, the predicted $q$-value depends on what holds a single body together (gravity or tensile strength) and on the mechanism which is driving the damping, i.e., catastrophic collisions, collisions with equal-sized bodies or with the smallest bodies in the system. For solids with sizes $\sim 1-10 \mathrm{~mm}$, which are held together by tensile strength, the predicted $q$ values are $3.65 \leqslant q<3.82, q=4, \quad 4 \leqslant q<4.33$ in the cases of catastrophic collisions, collisions with equal-sized bodies and with the smallest bodies, respectively (Pan \& Schlichting 2012).

Gaspar et al. (2012) investigated the effects of model parameters on the evolution of the solid size distribution using numerical models of collision-dominated debris disks. Their calculations yield a quasi steady-state slope of $q=3.65$, with a range between $q \approx 3.51-3.82$ when considering large variations for their model parameters.

When comparing these theoretical predictions with the results of our analysis, the classic prediction of the Dohnanyi (1969) collisional cascade lies at $\approx 3 \sigma$ from the weighted mean of the $q$-values in our sample of disks (see Figure 3). The lowest values predicted by the Gaspar et al. (2012) models are very similar to the Dohnanyi (1969) prediction, and therefore in line with the results for our debris disks, although these are obtained only with very large scaling factors for the strength curve, i.e., $S \gg 10^{8} \mathrm{erg} \mathrm{g}^{-1}$ (Benz \& Asphaug 1999).

Instead, the models by Pan \& Schlichting (2012) predict values which are significantly larger than our results (Figure 3 ). This may imply that the condition of exact balance between viscous stirring and some damping mechanism, which is the key assumption in those models, is not met in real disks.

A possible way to alleviate the apparent discrepancy of these findings with the model predictions is that part of the emission detected at $6.8 \mathrm{~mm}$ may not come from dust. If this was the case, our estimates would underestimate the true spectral index of the dust emission and therefore the $q$-value as well. For example, in the case of the AU Mic star, the emission seen by ALMA at $1.3 \mathrm{~mm}$ from the central regions of the debris disk can be explained by models of $\sim 10^{6} \mathrm{~K}$ plasma in an active stellar corona, which can also reproduce the strong X-ray flux from this source (Cranmer et al. 2013; MacGregor et al. 2013). 
Table 3

Results of the Analysis

\begin{tabular}{|c|c|c|c|c|c|c|c|c|}
\hline Source & $\begin{array}{l}\text { Sub-mm/mm Wavelength } \\
(\mathrm{mm})\end{array}$ & $\begin{array}{l}\text { Flux Density } \\
\qquad(\mathrm{mJy})\end{array}$ & Reference & $\alpha_{\mathrm{mm}}$ & $\begin{array}{c}T_{\text {dust }} \\
(\mathrm{K})\end{array}$ & Reference & $\alpha_{\text {Planck }}$ & $q$ \\
\hline$q^{1}$ Eri & 0.35 & $179.9 \pm 14.6$ & 1 & $2.55 \pm 0.07$ & $49 \pm 10$ & 6 & $1.86_{-0.04}^{+0.02}$ & $3.38 \pm 0.07$ \\
\hline HD 107146 & 1.25 & $12.5 \pm 1.3$ & 2 & $2.55 \pm 0.11$ & $29_{-5}^{+23}$ & 2 & $1.90_{-0.02}^{+0.05}$ & $3.36_{-0.08}^{+0.07}$ \\
\hline HD 181327 & 0.87 & $51.7 \pm 6.2$ & 3 & $2.86 \pm 0.09$ & $82 \pm 10$ & 7 & $1.96 \pm 0.01$ & $3.50 \pm 0.07$ \\
\hline HD 95086 & 0.35 & $112 \pm 16$ & 4 & $2.53 \pm 0.10$ & $55 \pm 10$ & 4 & $1.87_{-0.03}^{+0.02}$ & $3.37 \pm 0.07$ \\
\hline$\beta \mathrm{Pic}$ & 0.87 & $60 \pm 6^{\mathrm{a}}$ & 5 & $2.81 \pm 0.10$ & $85 \pm 10$ & 5 & $1.96 \pm 0.01$ & $3.47 \pm 0.08$ \\
\hline
\end{tabular}

Notes.

${ }^{a}$ Observations of $\beta$ Pic are affected by spatial filtering (see text for more details).

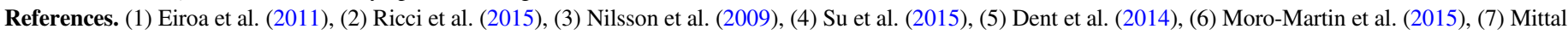
et al. (2015).
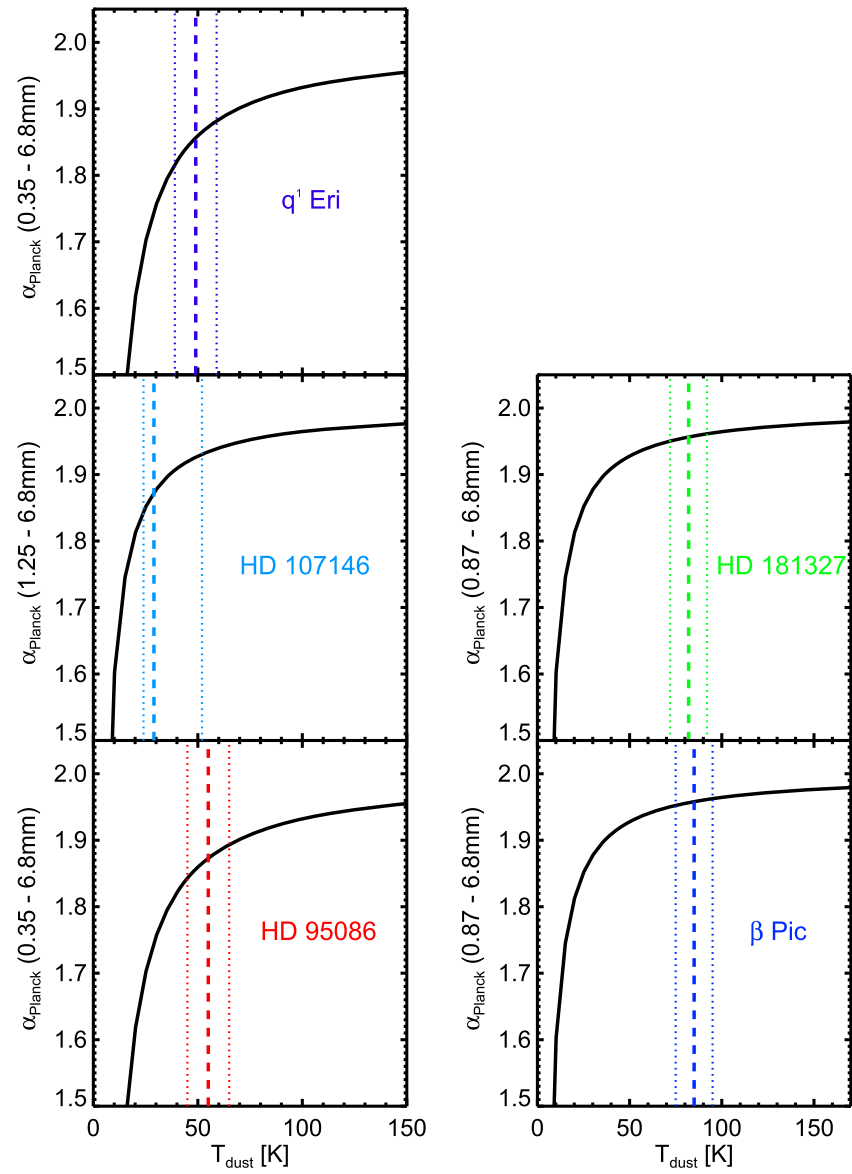

Figure 2. Spectral index of the Planck function as a function of dust temperature. In each panel, the dashed vertical line shows the dust temperature adopted in our analysis, and the dotted vertical lines show the limiting values of the intervals reported in Table 3.

For HD 107146, which shows the lowest inferred $q$-value among the debris disks in our sample, $\approx 65 \%$ of the measured flux at $6.8 \mathrm{~mm}$ would have to be produced by ionized gas to give a slope of the solid size distribution of $\approx 3.70$, which would be more in line with the predictions of the Pan \& Schlichting (2012) and Gaspar et al. (2012) models. However, the ALMA spatially resolved map for this disk at $1.25 \mathrm{~mm}$ does not show any hint for emission from the star location, as one would expect for emission from plasma in the stellar corona. The same can be argued for the spatially resolved ATCA maps at $6.8 \mathrm{~mm}$ for Fomalhaut (Ricci et al. 2012) and $\beta$ Pic

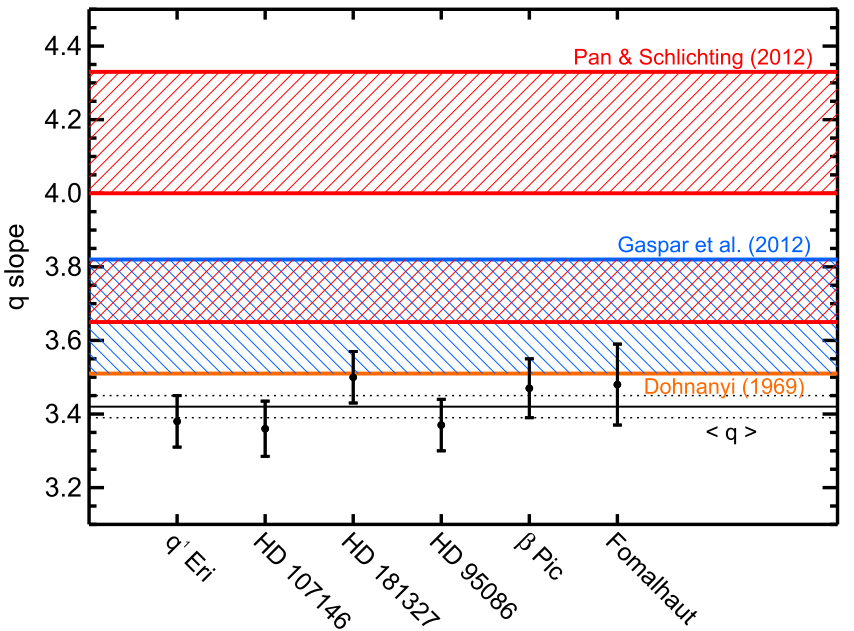

Figure 3. Inferred $q$-values of the slope of the solid size distribution for our sample of debris disks together with model predictions. Data-points show the values for the disks in our sample, including Fomalhaut from Ricci et al. (2012). The solid and dotted black horizontal lines show the weighted mean and uncertainty, i.e., $3.42 \pm 0.03$. The predictions from the different classes of models are indicated with the different colors. In particular, the horizontal orange line shows the single-valued classical (Dohnanyi 1969) prediction, the blue horizontal band with oblique blue lines represent the Gaspar et al. (2012) predictions, while the two red horizontal bands with oblique red lines show the values predicted by Pan \& Schlichting (2012, see discussion in Section 6).

(Figure 1), which do not show any strong emission from the stellar location.

For the disks which have not been yet spatially resolved at millimeter wavelengths, observations with higher angular resolution are needed to investigate possible ionized gas emission from the stellar corona. Also, observations at even longer wavelengths in the radio have the ability to disentangle the contribution of dust and ionized gas from the radio spectrum.

\section{SUMMARY}

We have presented new ATCA observations for the continuum emission at $6.8 \mathrm{~mm}$ of five debris disks, i.e., $q^{1}$ Eri, HD 107146, HD 181327, HD 95086, $\beta$ Pic. By combining these new detections with those at shorter sub-mm/mm wavelengths we have measured the sub- $\mathrm{mm} / \mathrm{mm}$ spectral index for these sources.

At these long wavelengths the dust emission is close to the Rayleigh-Jeans approximation, and we used previous estimates for the dust temperature in each of these systems to quantify the 
deviation from the Rayleigh-Jeans regime. We derived the spectral index $\beta$ of the dust emissivity $\left(\kappa_{\nu} \propto \nu^{\beta}\right)$, and used a relation derived by Draine (2006) to infer the slope $q$ of the solid size distribution $\left(n(a) \propto a^{-q}\right)$.

The slopes we derived range between $\approx 3.36$ and 3.50 with values consistent within the errorbars and a weighted mean $\langle q\rangle=3.42 \pm 0.03$. We compared these findings with the predictions of models of collisional cascades in debris disks. Models by Pan \& Schlichting (2012), which assume a balance between viscous stirring and some damping mechanism, overpredict these estimates significantly. Numerical models by Gaspar et al. (2012) can predict values as low as $\approx 3.5$, but only by invoking very large scaling factors for the strength curve $\left(S \gg 10^{8} \mathrm{erg} \mathrm{g}^{-1}\right)$. With a prediction of 3.51 , the classical models of Dohnanyi (1969) appear to be the closest to reproduce our results. This may indicate that the variation of the solid velocity dispersions and/or collision parameters are weaker than previously thought.

\section{REFERENCES}

Ardila, D. R., Golimowski, D. A., Krist, J. E., et al. 2004, ApJ, 617L, 147 Benz, W., \& Asphaug, E. 1999, Icar, 214, 179

Bonfanti, A., Ortolani, S., Piotto, G., \& Nascimbeni, V. 2015, A\&A, 575, 18

Butler, R. P., Wright, J. T., Marcy, G. W., et al. 2006, ApJ, 646, 505

Casagrande, L., Schoenrich, R., Asplund, M., et al. 2011, A\&A, 530, 138

Chen, C. H., Sargent, B. A., Bohac, C., et al. 2006, ApJS, 166, 351

Corder, S., Carpenter, J. M., Sargent, A. I., et al. 2009, ApJ, 690L, 65

Cranmer, S. R., Wilner, D. J., \& MacGregor, M. A. 2013, ApJ, 772, 149

Dent, W. R. F., Wyatt, M. C., Roberge, A., et al. 2014, Sci, 343, 1490

de Zeeuw, P. T., Hoogerwerf, R., de Bruijne, J. H. J., Brown, A. G. A., \&

Blaauw, A. 1999, AJ, 117, 354

Dohnanyi, J. S. 1969, JGR, 74, 2531

Draine, B. T. 2006, ApJ, 636, 1114

Durda, D. D., \& Dermott, S. F. 1997, Icar, 130, 140
Eiroa, C., Marshall, J. P., Mora, A., et al. 2011, A\&A, 555, 11

Ertel, S., Wolf, S., Metchev, S., et al. 2011, A\&A, 533, 132

Gaspar, A., Psaltis, D., Rieke, G. H., \& Ozel, F. 2012, ApJ, 754, 74

Gray, R. O., Corbally, C. J., Garrison, R. F., et al. 2006, AJ, 132, 161

Hughes, A. M., Wilner, D. J., Andrews, S. M., et al. 2011, ApJ, 740, 38

Kenyon, S. J., \& Bromley, B. C. 2015, ApJ, 806, 42

Lagrange, A.-M., Bonnefoy, M., Chauvin, G., et al. 2010, Sci, 329, 57

Liseau, R., Risacher, C., Brandeker, A., et al. 2008, A\&A, 480, 47

MacGregor, M. A., Wilner, D. J., Rosenfeld, K. A., et al. 2013, ApJ, 762L, 21

Mamajek, E. E., \& Bell, C. P. M. 2014, MNRAS, 445, 2169

Matthews, B. C., Kennedy, G., Sibthorpe, B., et al. 2014, in Protostars \&

Planets VI, ed. H. Beuther et al. (Tucson, AZ: Univ. Arizona Press), 521

Mittal, T., Chen, C. H., Jang-Condell, H., et al. 2015, ApJ, 798, 87

Moor, A., Abraham, P., Derekas, A., et al. 2006, ApJ, 644, 525

Morales, F. Y., Rieke, G. H., Werner, M. W., et al. 2011, ApJL, 730, L29

Moro-Martin, A., Marshall, J. P., Kennedy, G., et al. 2015, ApJ, 801, 143

Mustill, A., \& Wyatt, M. 2009, MNRAS, 399, 1403

Nilsson, R., Liseau, R., Brandeker, A., et al. 2009, A\&A, 508, 1057

Pan, M., \& Schlichting, H. E. 2012, ApJ, 747, 113

Rameau, J., Chauvin, G., Lagrange, A.-M., et al. 2013, ApJL, 772, L15

Ricci, L., Carpenter, J. M., Fu, B., et al. 2015, ApJ, 798, 124

Ricci, L., Testi, L., Maddison, S. T., \& Wilner, D. J. 2012, A\&A, 539L, 6

Schneider, G., Grady, C. A., Hines, D. C., et al. 2014, AJ, 148, 59

Schneider, G., Silverstone, M. D., Hines, D. C., et al. 2006, ApJ, 650, 414

Silverstone, M. D. 2000, PhD thesis, Univ. California

Smith, B. A., \& Terrile, R. J. 1984, Sci, 226, 1421

Su, K. Y. L., Morrison, S., Malhotra, R., et al. 2015, ApJ, 799, 146

Testi, L., Birnstiel, T., Ricci, L., et al. 2014, in Protostars \& Planets VI, ed.

H. Beuther et al. (Tucson, AZ: Univ. Arizona Press), 339

Trilling, D. E., Bryden, G., Beichman, C. A., et al. 2008, ApJ, 674, 1086

Ubach, C., Maddison, S. T., Wright, C. M., et al. 2012, MNRAS, 425, 3137

van Leeuwen, F. 2007, A\&A, 474, 653

Vidal-Madjar, A., Lagrange-Henri, A.-M., Feldman, P. D., et al. 1994, A\&A, 290, 245

Williams, J. P., Najita, J., Liu, M. C., et al. 2004, ApJ, 604, 414

Wilner, D. J., Andrews, S. M., \& Hughes, A. M. 2011, ApJL, 727, 42

Wilson, W. E., Ferris, R. H., Axtens, P., et al. 2011, MNRAS, 416, 832

Wyatt, M. C. 2008, ARA\&A, 46, 339

Zuckerman, B. 2001, ARA\&A, 39, 549

Zuckerman, B., \& Song, I. 2004, ApJ, 603, 738 